\title{
Chinese Classical Prescription(Liang-Xue-Di-Huang Decoction)for Hemohhhoidal Disease:study protocol for a randomized controlled trial
}

qing zhou ( $\sim 13601401869 @ 163 . c o m$ )

Nanjing university of chinese medicine https://orcid.org/0000-0002-5432-3652

Shuo-yang Shi

Nanjing University of Chinese Medicine

Zong-qi He

Nanjing University of Chinese Medicine

Cheng-biao Xu

Nanjing University of Chinese Medicine

Ji Geng

Nanjing University of Chinese Medicine

Tuo Chen

Nanjing University of Chinese Medicine

Zhao-feng Shen

Nanjing University of Chinese Medicine

Dan Zhang

Nanjing University of Chinese Medicine

Feng Jiang

Nanjing University of Chinese Medicine

Yu-Gen Chen

Nanjing University of Chinese Medicine

Ben-sheng Wu

Nanjing University of Chinese Medicine

\section{Study protocol}

Keywords: Hemorrhoidal disease, Liang-Xue-Di-Huang Decoction, Chinese classical prescription, Randomized controlled trial

Posted Date: January 6th, 2020

DOI: https://doi.org/10.21203/rs.2.13478/v2 
License: (c) (i) This work is licensed under a Creative Commons Attribution 4.0 International License. Read Full License 


\section{Abstract}

Background: Hemorrhoidal disease (HD) is one of the commonest proctologic condition in the general population. Medical therapy for HD has not been formally confirmed due to the inconsistent of results. Liang-Xue-Di-Huang Decoction, a kind of ancient Chinese classical prescription, has been used to treat HD from the 19th century in China. However, clinical research of Liang-Xue-Di-Huang Decoction in the treatment of HD is lack. We designed this study to evaluate the efficacy and safety of Liang-Xue-DiHuang Decoction in the treatment of HD. Methods/Design: A randomized, controlled, double blind, double-mimetic agent and multicenter trial to evaluate the efficacy and safety of Liang-Xue-Di-Huang Decoction is proposed. HD patients (stage $I, \varangle, \varangle$ ) will be randomly assigned into Liang-Xue-Di-Huang Decoction with the addition of Diosmine mimetic agent, or Diosmine with the addition of Liang-Xue-DiHuang Decoction mimetic agent. Patients will receive a 7-days treatments and a 7-days follow-up. The primary outcome measure is the French Bleeding Score in 7 and 14 days. The Secondary outcome measures are Goligher Prolapse Score and Quality-of-Life Score in 7 and 14 days. Discussion: This study will provide objective evidence to evaluate the efficacy and safety of Liang-Xue-Di-Huang Decoction in treatment of HD. Trial registration: Chinese Clinical Trial Registry. ChiCTR-1900022531.Registered 15 Apr 2019, http://www.chictr.org.cn/listbycreater.aspx.

\section{Introduction}

Hemorrhoidal disease (HD), one of the oldest and commonest proctologic condition in the general population, is more frequent in industrialized countries ${ }^{[1]}$. The true prevalence of $\mathrm{HD}$ in the general population is unknown and probably differents from country to country ${ }^{[2]}$. In the United States, HD is the third most common outpatient gastrointestinal diagnosis, affecting between 20 to $50 \%$ of the population and resulting in 4 million emergency visits annually ${ }^{[3]}$. Chronic bleeding is the main symptom of $\mathrm{HD}^{[4]}$.

Treatments for HD include medical therapies and surgery ${ }^{[5]}$. Medical therapies for HD have not been formally studied where the results have been inconsistent ${ }^{[3]}$. Increased fiber and fluid intake has been shown to improve symptoms of mild-to-moderate bleeding ${ }^{[6]}$. However, the fiber is not recommended as primary treatment to severe bleeding ${ }^{[7]}$. Another common medical prescription in patients with bleeding hemorrhoids is micronized purified flavonoid fraction. These drugs include diosmin, hesperidin, and cumarin. Each of these treatments has drawbacks, such as mild gastrointestinal disturbances ${ }^{[2]}$. There is still no evidence from well-designed studies to support the use of any of the over-the-counter preparations $^{[8]}$.

Liang-Xue-Di-Huang Decoction, a kind of Chinese herbal medicine, listed in Table 1, which has been approved by the National Administration of Traditional Chinese Medicine of People's Republic of China in $2018^{[9]}$. Liang-Xue-Di-Huang Decoction has been used for HD from the 19th century in China with good effects and few adverse events. However, it was still necessary to prove the efficacy and safety of this ancient Chinese classical prescription. The aim of the present study is to evaluate Liang-Xue-Di-Huang 
Decoction in the treatment of HD using a randomized, controlled, blind and multicenter trial among officially registered Chinese colorectal consultants, fellows and residents in China.

\section{Methods/design}

\section{Design}

This study is designed as a randomized, controlled, blind and multicenter trial. Trained researchers introduce the trial to patients, give them information sheets and consent forms. All patients have to obtain "Ethics approval and consent to participate" section and give their written informed consents prior to enrolment. The study's flow chart is shown in Figure 1.

\section{Ethics}

The trial protocol is conducted in accordance with the Good Clinical Practice Guidelines and the Declaration of Helsinki (2008) ${ }^{[10]}$. Central ethical approval has been confirmed from the group leader's ethic committee of Affiliated Hospital of Nanjing University of Chinese Medicine (ref approval no. 2019NL-158-02) and four sub-centers ethical will comply with the group leader's ethics approval. Written informed consent will be obtained from each patient.

\section{Recruitment}

A total of 240 Chinese patients who fulfill the screening criteria will be recruited at five hospitals in China: 1) Group leader, Affiliated Hospital of Nanjing University of Chinese Medicine, will recruit 64 patients through posters, 2) Sub-center, Changzhou Hospital of Traditional Chinese Medicine, will recruit 60 patients through posters, 3) Sub-center, Suzhou Hospital of Traditional Chinese Medicine, will recruit 60 patients through posters, 4) Sub-center, Xuyi Hospital of Traditional Chinese Medicine, will recruit 28 patients through posters, 5) Sub-center, Wujin Hospital of Traditional Chinese Medicine, will recruit 28 patients through posters.

\section{Sample size}

According to the literature search results, bleeding was used as the primary outcome measure for the treatment of HD by diosmin. It was estimated that Liang-Xue-Di-Huang Decoction treatment is not inferior to that of diosmin treatment. According to the non-inferiority test sample size calculation formula, for a two-sided significance level of 0.05 , the standard deviation is $0.4,(a=0.05, \beta=0.2, \delta=0.15)$, the noninferiority bound is 0.15 when the degree of grasp $(1-\beta)=80 \%$, the sample size is calculated using the formula: (See Formula 1 in the Supplemental Files)

Considering a $10 \%$ loss to follow-up, the sample size is 240 cases ( $n=120$ in each group).

\section{Randomization}


Block randomization was used. Stratification was carried out according to the center. With the help of SAS 9.4 statistical software, the random number table of central coding was generated for a given number of seeds. The subjects were randomly divided into Liang-Xue-Di-Huang Decoction with the addition of Diosmine mimetic agent, or Diosmine with the addition of Liang-Xue-Di-Huang Decoction mimetic agent. An independent person (Zhao-feng Shen), who is not involved in observation or assessment of the patients possesses the computer-generated randomization sequence.

The randomization procedure will be conducted by research assistants using an online computerized randomization system (https://sci.medroad.cn/).

\section{Blinding}

This trial is a double-blind trial, divided into the Liang-Xue-Di-Huang Decoction with the addition of Diosmine mimetic agent, or Diosmine with the addition of Liang-Xue-Di-Huang Decoction mimetic agent. The double levels of blinding are sealed separately. Treatments are blinded to the patients and investigators (including statisticians) until the entire study is completed. Each hospital receives an emergency letter, along with these test drugs, properly preserved until the end of the trial.

Code-breaking should occur only in the case of serious adverse events happen or further intervention of the patient needs to know the actual medication situation, with the permission of the person in charge of the research center, and a report should be submitted to the leader of the trial within 24 hours

\section{Eligibility criteria}

\section{Inclusion criteria:}

1.Comply with hemorrhoids standards diagnosis (stage $\mathrm{I}, \mathrm{\bigotimes}, \mathrm{\bigotimes})^{[2]}$;

2.Comply with "Traditional Chinese Medicine Disease and Syndrome, Diagnosis and Curative Effect Standard" damp-heat syndrome:bleeding hemorrhoids, bright red blood ${ }^{[11]}$;

3.Patient signed the informed consent form;

4. Patient agreed to avoid taking study agents outside the trial;

\section{Exclusion criteria:}

1.Patient had accompanied by severe liver, kidney, heart, brain, or lung dysfunction;

2.Patient had a history of inflammatory bowel disease, or a history of colorectal cancer, or had a history of any cancer;

4.Patient had a perianal abscess, anal fistula, rectal polyp, intestinal tumor or intestinal infectious disease; 
5.Patient will plan pregnancy during this study;

6.Patient is pregnant or lactating women at the time;

7.Patient was allergic to test drugs and their ingredients;

8.Patient had unability to understand the nature of the study and follow the doctor's recommendations.

9. Patient purchase or take other hemorrhoids medicine during this study period.

10. Patient had a history of bleeding disorders other than HD.

\section{Test drugs}

Test drugs include Liang-Xue-Di-Huang Decoction, Liang-Xue-Di-Huang Decoction mimetic agent, Diosmine and Diosmine mimetic agent. Liang-Xue-Di-Huang Decoction mimetic agent was consisted of

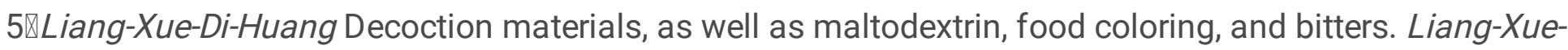
Di-Huang Decoction and Liang-Xue-Di-Huang Decoction mimetic agent are provided by Tianjiang Pharmaceutical Group Co. Ltd, Wuxi, China. Diosmine mimetic agent was consisted of $5 \rrbracket$ Diosmine materials, as well as maltodextrin, and food coloring. Diosmine and Diosmine mimetic agent are provided by NANJING CHIA TAI TIANQING Group Co. Ltd, Nanjing, China. All these mimetic agents have the same shape, size, taste, colour, package and Lot number.

\section{Interventions}

\section{Liang-Xue-Di-Huang Decoction with the addition of Diosmine mimetic agent}

Patients will take one Liang-Xue-Di-Huang Decoction per day, 2 times a day, be took 1 hour after lunch and dinner meals. Diosmine mimetic agent, $0.45 \mathrm{~g}$ each time, 2 times a day, be took 2 hours after lunch and dinner meals. The course of treatment will last 7 days, unless there is a loss of follow-up. Patients will be contacted by telephone in 7 and 14 days, and queried regarding adherence to study agents, illnesses, medication and supplement use. The assessment that needs to be performed at visit are listed in Figure 2.

\section{Diosmine with the addition of Liang-Xue-Di-Huang Decoction mimetic agent}

Diosmine with the addition of Liang-Xue-Di-Huang Decoction mimetic agent group's treatments and measurements will be in accordance with Liang-Xue-Di-Huang Decoction with the addition of Diosmine mimetic agent group.

\section{Outcome measures}

According to the latest treatment guidelines for $\mathrm{HD}^{[2]}$, the primary outcome measure of this study is the French Bleeding Score (Table 2) in 7 and 14 days. The Secondary outcome measure are Goligher 
Prolapse Score (Table 3) and Quality-of-Life Score (Table 4) in 7 and 14 days[available online at www.jvir.org].

\section{Emergency symptomatic treatment (serious bleeding)}

If patients have the following problems during this trial, such as a large amount of ejection bleeding accompanied by a significant decrease in hemoglobin, increased heart rate, and decreased blood pressure, patients will be discontinued and hospitalized.

\section{Adverse events}

All adverse events, including toxicity and side effects, such as gastrointestinal reaction, liver damage, and renal failure will be recorded and graded in detail throughout the study. When a severe adverse event occurs, researchers will provide every necessary treatment, and report the adverse event to the ethic committee of Affiliated Hospital of Nanjing University of Chinese Medicine.

\section{Safety evaluation}

A blood routine examination, routine urine test, liver function test, renal function test, electrocardiograph, and urine pregnancy test (women only) will be administered for safety outcomes, which are monitored both before and after clinical intervention.

\section{Data management}

Information from the clinical examination, as well as evaluation of treatment efficacy, will be recorded in each patient's case report form (CRF). The study record is the source document of clinical study subject and should be kept in group leader's hospital. Each center will design designated personnel to be the electronic CRF input staff. Upon completion of each subject observation, the investigator will promptly submit the study record to the CRF inputter. The electronic CRF encoder must review whether the project record of the study notes is complete and report on time.

\section{Data monitoring committee}

This clinical data monitoring committee was composed by Affiliated Hospital of Nanjing University of Chinese Medicine and Jiangsu Famous Medical Technology Co., Ltd, Nanjing, China.

\section{Patient's privacy protection}

Only researchers and arbitrator who will sign the confidentiality commitment in this clinical trial may come into contact with the participants' personal health records. Drug regulatory departments have the right to inspect the records of clinical trials. Data will be processed anonymously, and information identifying individual subjects will be omitted. Patient's medical records will be stored in group leader's data archives 


\section{Statistical analysis}

Frequency, median, and mean \pm standard deviation of the bleeding score, goligher prolapse score and quality-of-life score will be used for descriptive statistics. The statistical analysis will be performed using SAS 9.4. $P<0.05$ is considered statistically significant.

\section{Discussion}

$\mathrm{HD}$ is a well-defined clinical and pathophysiological placement between benign conditions but with high impact on quality of life ${ }^{[12]}$. Bleeding is the main symptom of $\mathrm{HD}^{[4]}$. It is estimated that $50 \%$ of people over 50 years of age have experienced symptoms of HD at least once in their life, and one-third of patients affected by HD seek medical attention ${ }^{[13,14]}$. Excessive bleeding can lead to an emergency situation. Hemorrhoidectomy and stapled hemorrhoidopexy are validated and effective surgical techniques, but are associated with long, painful postoperative courses. ${ }^{[15]}$. Contrary to common belief, a nonsurgical treatment is quite effective to manage $\mathrm{HD}^{[16]}$, which can be offered with expectations of minimal harm ${ }^{[6]}$. Therefore medical therapy should be the first-line therapy for this disease ${ }^{[17]}$.

Liang-Xue-Di-Huang Decoction has been used to treat HD from the 19th century in China and rich experience has been accumulated. In 2018, National Administration of Traditional Chinese Medicine of People's Republic of China published 100 classic prescriptions of ancient Chinese medicine, which contained Liang-Xue-Di-Huang Decoction ${ }^{[9]}$. These classic prescriptions of ancient Chinese medicine can be directly used in clinical treatment in China. In this classic Liang-Xue-Di-Huang Decoction, Sophora japonica L. (Huaijiao) and Platycladus orientalis(L.)Franco (Cebaiye) are the Jun (emperor) components. Sophora japonica L. (Huaijiao), which was first recorded in Sheng Nong's herbal classic, was commonly applied in clinical practice for the treatment of hematochezia from the 1th century in China. It has the effect of cooling blood, stopping bleeding, clearing heat in bowels and eliminating swell and easing pain $^{[18]}$. Modern pharmacological studies have demonstrated its efficacy for stopping bleeding and antiinflammation ${ }^{[19]}$. Platycladus orientalis(L.)Franco (Cebaiye) is categorized as a bloodcooling and hematostatic herb, which is usually prescribed with heat-clearing herbs to reinforce the efficacy of hemostasis. Sanguisorba officinalis L. (Diyu), Coptis chinensis Franch. (Huanglian), Rehmannia glutinosa Libosch. (Shengdihuang) are the Chen (minister) components, synergize with Jun to strengthen its therapeutic effects. In traditional Chinese medicine (TCM), Sanguisorba officinalis L. (Diyu) is often mixed with other herbs for the treatment of bleeding hemorrhoids. Coptis chinensis Franch. (Huanglian) has the effect of detumescence, clinically used for the treatment of hemorrhoid. Rehmannia glutinosa Libosch. (Shengdihuang) has been traditionally used as a blood cooling hemostatic. The Zuo (assistant) components, Angelica sinensis(oliv) Diels. (Danggui), Citrus aurantium L. (Zhike), Scutellaria baicalensis Georgi. (Huangqin), Paeonia lactiflora Pall. (Chishao), Schizonepeta tenuifolin Briq. (Jingjie), and Trichosanthes Kirilowii Maxim (Tianhuafeng), activate blood circulation to remove stasis, eliminate possible adverse effects of the Jun and/or Chen components. The Shi (courier) components, Cimicifuga heraclei folia.Kom (Shengma), and Glycyrrhiza uralensis Fisch. (Shenggancao) facilitate the overall 
action of the other components. Theoretically, Liang-Xue-Di-Huang Decoction work through the TCM therapeutic principle“Jun-Chen-Zuo-Shi”, to relieve bleeding hemorrhoids and diminish hemorrhoids prolapsed.

Although TCM has been clinically practised for thousands of years, most Chinese herbal medicine products do not possess up-to-date regarding about their safety and modern scientific evidences for their claimed clinical uses. TCM safety research is becoming more standardized and is gradually aligning with international standards. A randomized, controlled, blind, and multicenter trial will be helpful for further prove the efficacy and safety of Liang-Xue-Di-Huang Decoction as an treatment for HD.

\section{Abbreviation}

Hemorrhoidal disease $\quad$ HD

Case report form $\quad$ CRF

Traditional Chinese medicine TCM

\section{Declaration}

\section{Trial status}

The protocol version number is NO.2 and the date is January 10, 2019. At the time of manuscript submission, patient's recruitment for the trial is on-going. The clinical study will begin from Aug, 2019 and end in Dec, 2020. A total of 240 Chinese patients will be recruited in this clinical study.

\section{Funding}

This study is supported by the Key Laboratory of Famous Doctors' Proved Recipe Evaluation and Transformation Under State Administration of Traditional Chinese Medicine (NZYJDMF-2018002). This funding source provided test drugs, but it had no role in the design of this study and did not have any responsibility for analyses, interpretation of the data or the decision to submit results.

\section{Authors' contributions}

Qing Zhou and Shuo-yang Shi contributed to the design of the study protocol. Tuo Chen participated in the statistical design and helped in the design of the study. Ben-sheng Wu, Cheng-biao Xu, Ji Geng, Dan Zhang, Feng Jiang and Zhong-qi He helped to draft the manuscript and participated in the project development. Yu-Gen Chen was the project leader for this research and participated in the critical revision of the manuscript. All authors have read and approved the final manuscript.

\section{Competing interests}

None. 
Acknowledgements

Not applicable.

\section{Consent for publication}

Individual participant image or other clinical detail is not applicable.

\section{References}

[1] Jacobs DO.Hemorrhoids:what are the options in 2018?.Curr Opin Gastroenterol 2018,34:46-49.

[2] Altomare DF,Giuratrabocchetta S.Conservative and surgical treatment of haemorrhoids.Nat Rev Gastroenterol Hepatol 2013,10:513-521.

[3] Robert S,SandlerAnne F,Peery.Rethinking What We Know About Hemorrhoids CLIN GASTROENTEROL H 2019,17:8-15.

[4] G G Ravindranath,B G Rahul.Prevalence and risk factors of hemorrhoids:a study in a semi-urban centre.Int Surg J 2018,5:496-499.

[5] Wilson MZ,Swarup A,T Wilson LR,et al.The Effect of Nonoperative Management of Chronic Anal Fissure and Hemorrhoid Disease on Bowel Function Patient-Reported Outcomes.Dis Colon Rectum 2018,61:1223-1227.

[6] Davis BR,Lee-Kong SA,Migaly J,et al.The American Society of Colon and Rectal Surgeons Clinical Practice Guidelines for the Management of Hemorrhoids.Dis Colon Rectum 2018,61:284-292.

[7] Garg P.Conservative Treatment of Hemorrhoids Deserves More Attention in Guidelines and Clinical Practice.Dis Colon Rectum 2018,61:e348.

[8] ZagriadskiI EA,Bogomazov AM,Golovko EB.Conservative Treatment of Hemorrhoids:Results of an Observational Multicenter Study.Adv Ther 2018,35:1979-1992.

[9] National Administration of Traditional Chinese Medicine of People's Republic of China.Notice of National Administration of Traditional Chinese Medicine of People's Republic of China on the publication of the catalogue of 100 classic prescriptions of ancient Chinese medicine (the first batch) [EB/OL].http://kjs.satcm.gov.cn/zhengcewenjian/2018-04-16/7107.html

[10] Vijayananthan A,Nawawi O:The importance of Good Clinical Practice guidelines and its role in clinical trials.Biomed Imaging Interv J 2008,4:e5.

[11] State administration of traditional Chinese medicine.Criteria for diagnosis and efficacy of TCM diseases.Nanjing:nanjing university press 1994:132-133. 
[12] Rubbini M,Ascanelli S,Fabbian F.Hemorrhoidal disease: is it time for a new classification? Int J Colorectal Dis 2018,33:831-833.

[13] Garg P.Novel Nonsurgical Treatment of Intractable Bleeding in Hemorrhoid Patients on Anticoagulants.Surg Innov 2018,25:423.

[14] Shelygin Y,Krivokapic Z,Frolov SA,et al. Clinical acceptability study of micronized purified flavonoid fraction $1000 \mathrm{mg}$ tablets versus $500 \mathrm{mg}$ tablets in patients suffering acute hemorrhoidal disease. CURR MED RES OPIN 2016,32:1821-1826.

[15] Watson AJ,Hudson J,Wood J,et al.Comparison of stapled haemorrhoidopexy with traditional excisional surgery for haemorrhoidal disease (eTHoS):a pragmatic,multicentre,randomised controlled trial.Lancet 2016; 388:2375-2385.

[16] Garg P.Conservative Treatment of Hemorrhoids Deserves More Attention in Guidelines and Clinical Practice.Dis Colon Rectum 2018,61:e348.

[17] Wilson MZ,Swarup A,T Wilson LR,et al.The Effect of Nonoperative Management of Chronic Anal Fissure and Hemorrhoid Disease on Bowel Function Patient-Reported Outcomes.Dis Colon Rectum 2018,61:1223-1227.

[18] The State Pharmacopoeia Commission of PR China, Chinese Pharmacopoeia Commission, 2015 ed.,Beijing, 2015, http://www.drugfuture.com/Pharmacopoeia/CP2015-1/1642-1643.pdf.

[19] Shuang qin Wang,Jingjing Zhang,Juan Liu.et al.Quality evaluation of huaijiao pill by chromatographic fingerprint and simultaneous determination of its major bioactive components.Journal of Pharmaceutical Analysis 2016,4:249-255.

\section{Tables}

Table 1 Standard formulation of Liang-Xue-Di-Huang Decoction

\begin{tabular}{|c|c|c|c|c|c|}
\hline Pinyin name & Latin name & Doses & $\begin{array}{l}\text { Pinyin } \\
\text { name }\end{array}$ & Latin name & Doses \\
\hline Huaijiao & Sophora japonica L. & $9 g$ & Cebaiye & Platycladus & $6 g$ \\
\hline Diyu & Sanguisorba officinalis $L$. & $6 g$ & Huanglian & Coptis chinensis Franch. & $6 \mathrm{~g}$ \\
\hline Shengdihuang & $\begin{array}{l}\text { Rehmannia glutinosa } \\
\text { Libosch. }\end{array}$ & $6 g$ & Danggui & Angelica sinensis(oliv) Diels. & $4.5 \mathrm{~g}$ \\
\hline Zhike & Citrus aurantium $L$. & $3 g$ & Huangqin & $\begin{array}{l}\text { Scutellaria baicalensis } \\
\text { Georqi. }\end{array}$ & $3 g$ \\
\hline Chishao & Paeonia lactiflora Pall. & $3 g$ & Jingjie & $\begin{array}{l}\text { Schizonepeta tenuifolin } \\
\text { Briq. }\end{array}$ & $3 g$ \\
\hline Tianhuafeng & $\begin{array}{l}\text { Trichosanthes Kirilowii } \\
\text { Maxim. }\end{array}$ & $2.4 \mathrm{~g}$ & Shengma & $\begin{array}{l}\text { Cimicifuga heraclei } \\
\text { folia.Kom }\end{array}$ & $1.5 \mathrm{~g}$ \\
\hline Shenggancao & $\begin{array}{l}\text { Glycyrrhiza uralensis } \\
\text { Fisch. }\end{array}$ & $1.5 \mathrm{~g}$ & & & \\
\hline
\end{tabular}




\begin{tabular}{lc}
\hline Table 2 French Bleeding Score & (Possible Score=0-9) \\
\hline Frequency & 0 \\
Never & 1 \\
\hline$>1$ per year & 2 \\
\hline 1 per month & 3 \\
\hline 1 per week & 4 \\
\hline >1 per day or bowel movement & \\
\hline Bleeding & 0 \\
\hline Never & 1 \\
\hline At wiping & 2 \\
\hline In the toilet & 3 \\
\hline On underwear & 0 \\
\hline Anemia & 1 \\
\hline Never & 2 \\
\hline Without transfusion & \\
\hline With transfusion
\end{tabular}

\begin{tabular}{clc}
\hline Table 3 & Goligher Prolapse Score (Possible Score=0-3) \\
\hline Grade 1 & No protrusion & 0 \\
Grade 2 & Protrusion with spontaneous reduction & 1 \\
Grade 3 & Protrusion requiring manual reduction & 2 \\
Grade 4 & Protrusion that cannot be reduced & 3 \\
\hline
\end{tabular}

Table 4 Quality-of-Life Score (Possible Score=0-4)

No discomfort 0

Mild discomfort

Moderate discomfort 2

Significant discomfort 3

Permanent discomfort 4 


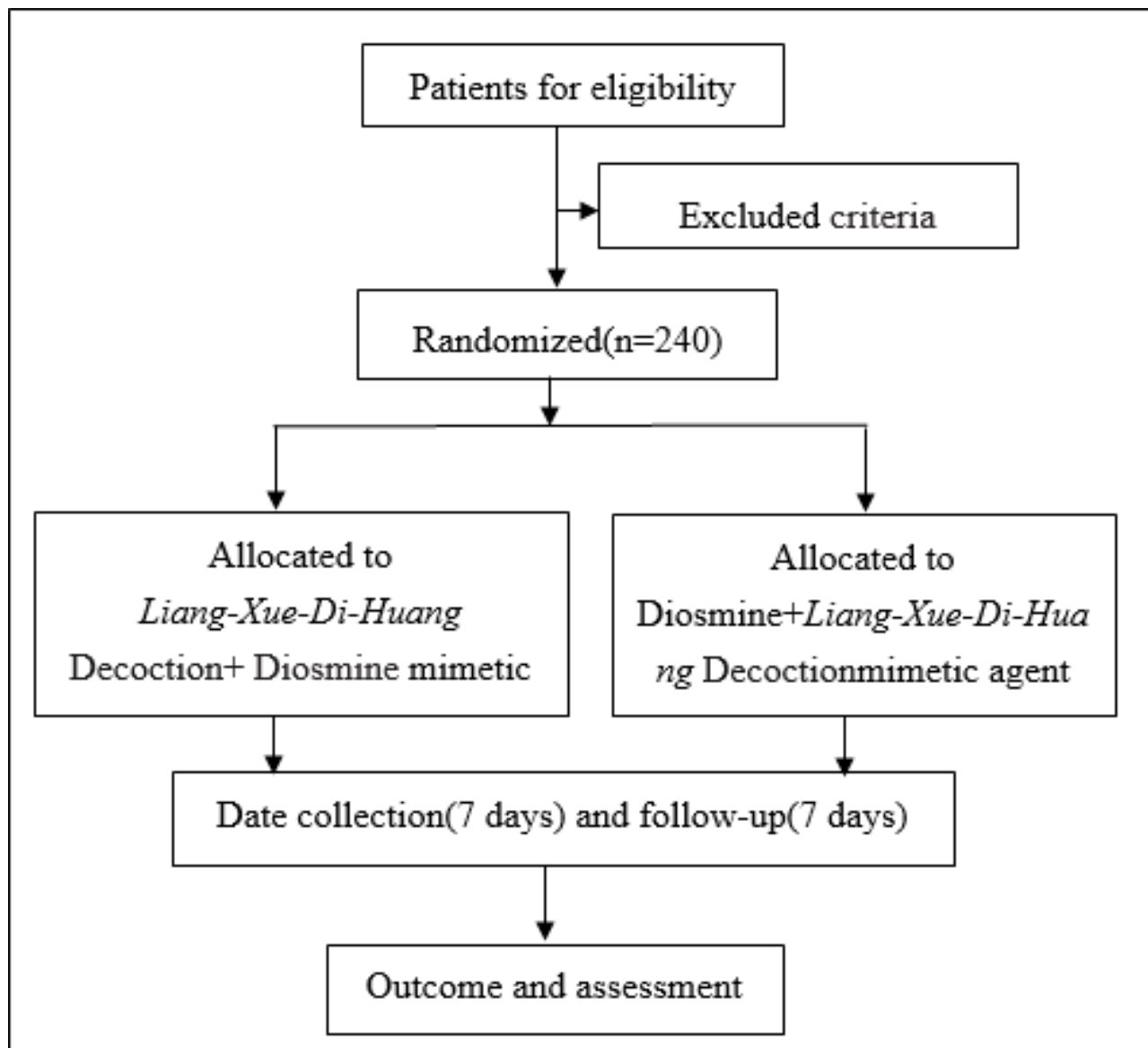

Figure 1 Study flow chart. The flow chart of enrolment,allocation, intervention and assessment.

\section{Figure 1}

Study flow chart. The flow chart of enrolment, allocation, intervention and assessment. 


\begin{tabular}{|c|c|c|c|c|c|}
\hline \multirow[b]{3}{*}{ Visit } & \multicolumn{5}{|c|}{ Study period } \\
\hline & \multirow{2}{*}{$\begin{array}{c}\text { Enrolment } \\
\text { Visit } 1\end{array}$} & \multirow{2}{*}{$\frac{\text { allocation }}{\text { Visit } 2}$} & \multicolumn{2}{|c|}{ Post-allocation } & \multirow{2}{*}{$\begin{array}{c}\text { Close-out } \\
\text { Visit } 4\end{array}$} \\
\hline & & & & Visit 3 & \\
\hline Time point & Day -1 & Day 0 & Dayl & Day 7 & Day 14 \\
\hline \multicolumn{6}{|l|}{ Enrollment } \\
\hline Informed consent form & $\bullet$ & & & & \\
\hline History & $\bullet$ & & & & \\
\hline Age & $\bullet$ & & & & \\
\hline Gender & $\bullet$ & & & & \\
\hline Inclusion criteria & $\bullet$ & & & & \\
\hline Exclusion criteria & $\bullet$ & & & & \\
\hline Randomigation and allocation & $\bullet$ & & & & \\
\hline Drug distribution & & - & & & \\
\hline \multicolumn{6}{|l|}{ Intervention } \\
\hline $\begin{array}{l}\text { Liang-Xue-Di-Huang Decoction } \\
\text { + Diosmine mimetic agent }\end{array}$ & & & $\bullet$ & $\bullet$ & \\
\hline $\begin{array}{l}\text { Diosmine } \\
\text { + Liang-Xue-Di-Huang } \\
\text { Decoction mimetic agent }\end{array}$ & & & $\bullet$ & $\bullet$ & \\
\hline \multicolumn{6}{|l|}{ Assessme } \\
\hline Ital signs & $\bullet$ & & & $\bullet$ & $\bullet$ \\
\hline Physical examination & $\bullet$ & & & $\bullet$ & $\bullet$ \\
\hline Blood routine examination & $\bullet$ & & & $\bullet$ & $\bullet$ \\
\hline Routine urine test & $\bullet$ & & & $\bullet$ & $\bullet$ \\
\hline Electrocardiograph & $\bullet$ & & & $\bullet$ & $\bullet$ \\
\hline Liver function test & $\bullet$ & & & $\bullet$ & $\bullet$ \\
\hline Renal function test & $\bullet$ & & & $\bullet$ & $\bullet$ \\
\hline Proctoscopy & $\bullet$ & & & $\bullet$ & $\bullet$ \\
\hline Urine pregnancy test (women) & $\bullet$ & & & $\bullet$ & $\bullet$ \\
\hline Bleeding score & $\bullet$ & & & $\bullet$ & $\bullet$ \\
\hline Goligher prolapse score & $\bullet$ & & & $\bullet$ & $\bullet$ \\
\hline Quality-of-life score & $\bullet$ & & & $\bullet$ & $\bullet$ \\
\hline
\end{tabular}

Figure 2 Study schedule for patients. After the enrolment and allocation, participants will receive a 7-days treatments and a 7-days follow-up. The time-points of assessment are shown in the schedule.

\section{Figure 2}

Study schedule for patients. After the enrolment and allocation, participants will receive a 7-days treatment and a 7-days follow-up. The time-points of assessment are shown in the schedule.

\section{Supplementary Files}


This is a list of supplementary files associated with this preprint. Click to download.

- Formula1.jpg

- 20191229SPIRITChecklist.docx 